\title{
Deployment Schemes in WSNs
}

\author{
Yang $\mathrm{Li}^{1}$, N. Thirupathi Rao ${ }^{2}$, Debnath Bhattacharyya ${ }^{3}$ \\ ${ }^{1}$ Harbin University of Science and Technology, China \\ ${ }^{2,3}$ Department of Computer Science and Engineering, Vignan's Institute of \\ Information Technology (A), Visakhapatnam, AP, India, \\ 1blueskyang@126.com, 2nakkathiru@gmail.com, ${ }^{3}$ debnathb@gmail.com
}

\begin{abstract}
Wireless sensor networks are gaining importance for the utilization in various scenarios day by day. The impact of utilizing these sensor networks is asking an important note on the day to day available issues in the human life. The working of such people is heavily impacted by these sensor nodes and their impact of working of them. When the nodes are working, they will collect the data from time to time and data will be processed by the machines and the required steps will be taken for the better processing of such data and for better utilization of the resources of devices or several other points to be considered for better utilization. Several models of sensor networks are available for the users or the developers such that to form the various good results. To achieve such good results, the deployment of nodes at various locations with various resources was very important. Hence, in the current article an attempt has been made to present a detailed review on various deployment schemes of nodes in the wireless sensor networks are discussed in detail.
\end{abstract}

Keywords: Sensors, nodes, networks, sensor networks, wireless units, wireless sensors, data collection, analysis of data, deployment, schemes, data analysis, deployment schemes.

\section{Introduction}

Professor Jerry Kaplan of Stanford University, an expert in artificial intelligence,"The development of AI will destroy most of the current human work,"'We can not avoid mass un Usage of WSNs is generally reasonable to an assortment of fields. Its usage is radically expanded on littler hubs and radio handset. The remote sensor systems finish its activity in autonomous form to obtain exact esteems in the network areas. The major role or the major part of these networks is the sensors that collect the data from various sources and the same will be transferred to the networks for further processing of such data. Several forma of the data will be collected from these sensor nodes placed at various locations. In the system, WSNs is a relatively novel request which provide excellent observing with nearly temperate gear for large geological zones [1]. WSNs comprised of a set of minor sensor hubs, by which neighboring condition can productively screen. In both academic and mechanical areas in present being WSNs have pulled in contingent more consideration as a result of it is across the board potential uses in combat zone supervision, ecological observing, climate estimating, human services and cataclysm acknowledgement and so on [2].

\subsection{LOCALIZATION OF WSN NODES}

Article history:

Received (May 29, 2019), Review Result (August 13, 2019), Accepted (September 20, 2019) 
The limitation is a procedure to register the areas of remote gadgets in a system WSN Composed of many hubs that are thickly sent in a district of interests to quantify sure wonder. None-observable pathway (NLOS) condition is the overwhelming components that influence confinement, which happens when a few impediments limit the immediate way from the obscure hub to the grapple hubs. Stay hubs are those hubs whose area should be known. There is an expansive scope of techniques for development of the NLOS affect on confinement precision. The area gauges from various gatherings are joint by utilising remaining weighting.

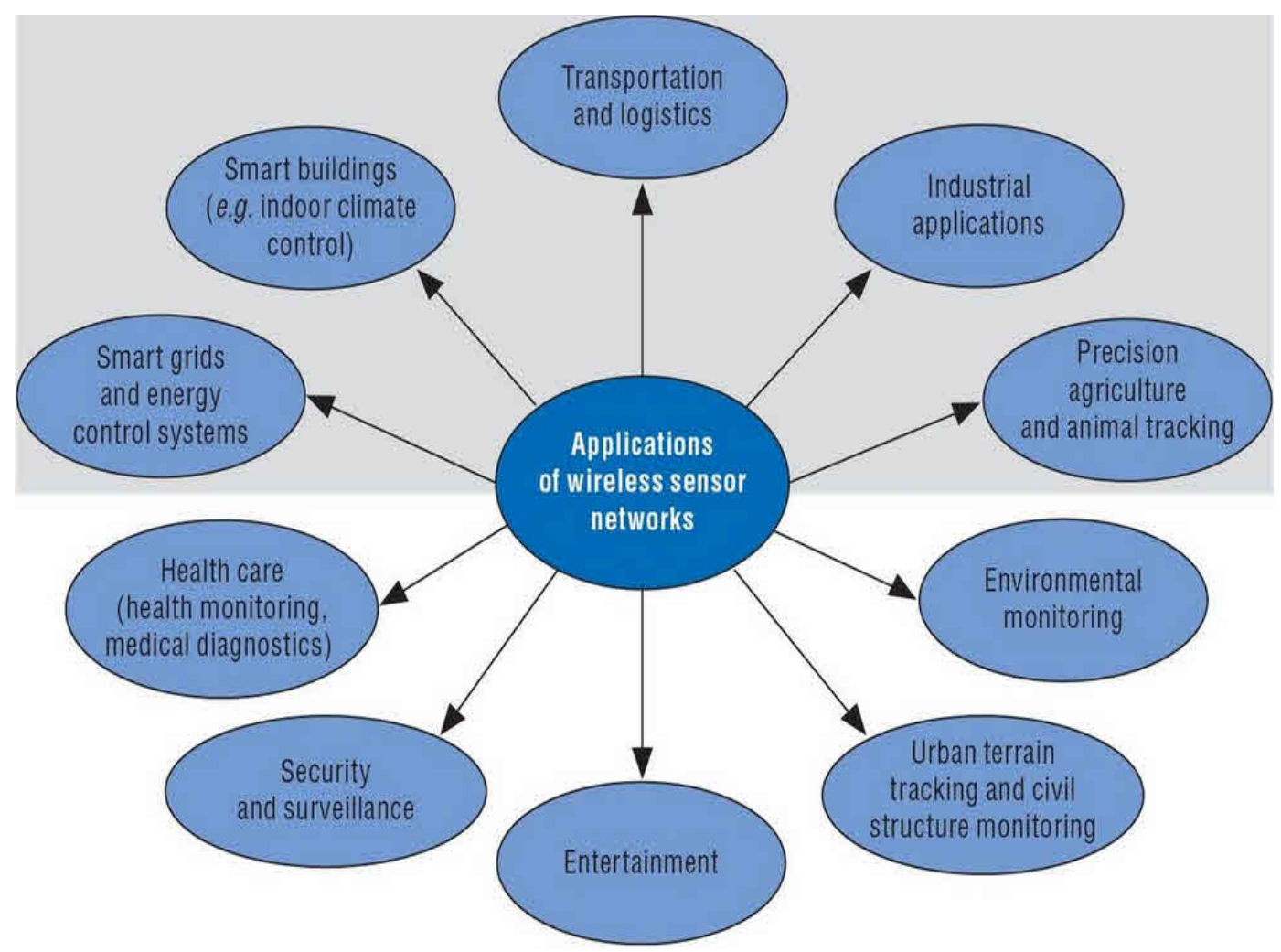

Figure 1. Applications of WSNs [2]

The theory testing is utilised in[3] to recognize the LOS and NLOS conditions, and after that, an expanded Kalman channel utilised as a nonlinear estimation. Acquiring data about the situation of sensors in remote systems (WSNs) is vital since it is the required for various assignments and these are target following, topographical directing conventions, ecological observing, and so forth. To diminish NLOS mistakes, the compelled enhancement strategies are utilised [4][3].

\section{LITERATURE REVIEW}

V. Karthik in 2012 [1] discussed in detail about the hub increments because of persistently sending of detected information from their own tyke hubs. Once the power in the parent hubs depleted, from the sink hub, a portion of the youngster hubs get segregated. The assessed information gathering strategy includes organisation of many versatile robots whose obligation is to gather the information from the hubs whose vitality level is not as much as the limit esteem. By utilising time and area, based systems Navigation of portable robots to gather the 
information from parceled hubs accomplished. In assessed cross breed planning, the route of versatile robots customized by both the mix of time and area-based procedures with a scope of district booking. In arrange situation, because of its additional duties, the versatile robot gets more weights to visit every single partitioned hub. So the whole situation is separated into different areas, and the sending of successive portable robots transferred to the necessities [5][6]. WSN is upgraded doubly utilize many versatile robots. As a result, the effectiveness of detected information gathered by the base station or sink hub from apportioned. The result from different angles demonstrates that anticipated various portable robots can build up the presence of gathering the detected information. The information was in colossal scale detecting fields.

M. Vijayalakshmi et al. in 2013 [2] augments and organises life and accomplish steadiness. To control expectation, examine the execution tradeoff between lessening correspondence cost and forecast cost, and plan calculations a clever plan utilised and it is utilised to take the benefit of versatile technique to allow/incapacitate estimate tasks. Over the first double forecast plot, Localized expectation strategy performed which is utilised to diminish correspondence and calculation cost by limiting the power usage. A rational calculation expected for information conglomeration will utilize quicker and fit group to-bunch engendering.

Nasser Aghaieet. al.in 2016 [3] in the field of remote sensor systems (WSN) Localization is one of the significant issues. A flag which is utilized to set up the separation between hubs can't go amid a straight way in the non-viewable pathway (NLOS) situations in light of the obstructions between the grapple hubs and different hubs. Because of This issue restriction blunder increments. Restriction technique based AOA estimation for the NLOS situations is displayed by creator. This technique in view of the distinguishing proof of NLOS hubs and afterwards dispensing with them from the limitation procedure.

Renuka .R. Patil et al. in 2015 [5] had utilized these networks as a part of the application zones name as, vehicle following, agribusiness, military, backwoods observation, social insurance, condition and seismic tremor assessment and so forth. To screen the natural framework based on utilizations of WSN These sensor hubs are to be conveyed in a specific area. An improvement and application necessity underpins. The multifaceted nature of organisation of remote sensor systems. The correlation of calculations and conventions is finished considering parameters like vitality utilization; scope of hubs, normal separation between the hubs and so on. And chooses the better execution sending calculation. This paper gives the learning about the best of static, dynamic and vitality mindful organisation plans.

Po-Jen Chuang et al. in 2014 [6] Wireless sensor arranges consistently discover the area of an obscure hub by figuring the separation between the obscure hub and its neighbouring grapples. In this paper to improve both limitation exactness and confinement achievement rates, the creators present another neural system based hub restriction conspire. This plan make the prepared system show totally identified with the topology by means of web based preparing and corresponded topology-prepared information and in this manner accomplish between hub separate estimation and furthermore more proficient use of the neural systems. It likewise, to enhance the separation estimation exactness and also restriction precision at no extra cost embrace both got flag quality sign and bounce tallies to gauge the between hub separations. Trial assessment comes about demonstrate that, the new plan always creates higher confinement achievement rates and littler restriction blunders than different arrangements at sensible cost.

Annie UthraRajan et al. in 2015 [7] in businesses, condition observing and medicinal services checking frameworks remote sensor systems have turned into a developing innovation. Moreover, sensor hub regarding memory, data transfer capacity and vitality turn into an asset obliged gadget. These limitations in view of retransmission put in constrain clog in the system, 
gives extensive number of bundle drops, low throughput and vital wastage of energy. Another approach for foreseeing blockage utilising probabilistic technique, and overseeing clog utilising new rate control strategies is anticipated. The probabilistic technique utilised for the forecast of congestion in a hub is produced utilising actualities movement and cushion inhabitance. To enhance throughput and to diminish parcel drops the rate control technique utilises rate distribution plans, specifically, (RR) rate lessening, rate direction (RRG) and split convention (SP). A vitality productive steering which finds the finest sending hub for information. On examination of Simulation comes about with decentralized prescient blockage control demonstrate that they chose technique without a doubt limits clog and vitality utilization, and enhances the execution.

Rajesh M et al. in 2015 [8] proposed the organisation of sensor hubs by numerous independent versatile robots in an unexplored tremendous catastrophe inclined an area. The utilization of numerous robots gives huge points of interest over human-helped arrangement like wellbeing, exact situating and adaptability. For exact area of an occasion, confinement of the sensor hubs is essential which is accomplished by utilising RSS from stay hubs and the sensor hubs which go about as secure hub subsequent to being situated. Arrangement of hub by robots helps in accomplishing the area data of the considerable number of hubs that development the system. Correspondence and coordination between the numerous robots over the sensor hubs is utilised to achieve exact confinement, speedier investigation and system creation. In this investigation, vitality productive usage of the sensor hub is achieved when it goes about as a grapple hub as it just reacts when it gets the hub finds summon with a property of Zigbee convention from the robot putting the sensor hubs.

ShilpaMahajan et al. in 2015 [9]discussed about when information sends on a solitary path a few times it brings about consumption of vitality and henceforth crash of those hubs. Accordingly, mistake resilience technique for finding numerous disjoint courses for information. In this technique, the framework can transform from a blocked off way with broken connects to existing competitor ways. Another chart hypothesis plot for ideal way determination in view of nature of administration parameters is anticipated. A blame tolerant instrument is likewise embraced to draw out the life expectancy of the system. What's more, the outcomes demonstrate that the arranged approach boosts organise lifetime and way security makes strides.

Vijay S. Rao et al. in 2015[11]discussed about the measure of vitality gathered fluctuates spatially and transiently with the goal that the sensor hubs frequently don't have adequate energy to deal with application like system and house-keeping assignments. In addition the surrounding source can't be understood to be essentially accessible constantly. It is alluring that the hubs take up higher burdens at whatever point more vitality is reaped vitality. At the point when the vitality isn't adequate the hubs should change to profoundly vitality able plans. Thus necessity of gathering mindful planning of assignments emerges. Gathering mindful planning challenges are (a) to discover the measure of energy to be depleted in a scheduled opening, and (b) to devour this vitality for completing assignments maximally. Given the multifaceted nature of the MDP cannot be followed progressively, that is the reason a voracious planning arrangement had completed.

Tiago Semprebomy et al. in 2015 [10] blocking upkeep untrustworthy or challenging to reach situations. Making the system adaptable to disappointment and natural changes, excess arrangement procedures typically considered in this situation. Moreover, while dynamic hubs execute checking administrations, rest booking techniques can likewise be connected, empowering excess hubs to kill their radios. 


\section{DESIGN DIFFICULTIES AND DEPLOYMENT SCHEMES IN WSN}

In WSNs, to expand the lifetime of the entire system is the central plan challenges while considering the cost, vitality utilization and consistent quality. System lifetime can be expanded by considering heaps of variables that are a design of system and conventions, information accumulation, sensor hub lifetimes, channel qualities and vitality utilization demonstrate. One way to deal with augment the system life can expand through a vitality proficient dependable steering calculation which is for information correspondences inside WSNs, and this calculation can give the best outcomes by joining the aggregate vitality utilization, correspondence consistent quality and cost [4][7][8].

\subsection{NODE DEPLOYMENT}

The sensor hubs settled in the specific territory in static hub organisation, because of which the effective execution diminishes. In any case, if there should arise an occurrence of dynamic hub sending the execution expanded on account of sensor hubs is versatile. In vitality mindful hub organizations each sensor hub is outfitted with the power and is utilised for the primary accumulate and afterwards exchanged the information. For the transmission of information what measure of vitality is devoured by a hub is additionally characterised in this plan.

\subsection{ESSENTIAL TARGET O/THE HUB SENDING}

Sensors ought to be sent in such a way in this way, to the point that they convey in line to the general plan targets. Consequently, those organisation systems are in the writing which gives weight on expanding the scope region, upgrading the vitality utilization and furthermore accomplishing the strong system association, considers broadening the lifetime of the system or potentially expanding information devotion.

\subsection{DYNAMIC NODE DEPLOYMENT ALGORITHMS}

Sending calculation has pulled in researchers' deep consideration. To discover the places of the sensor hubs is the massive piece of sending, which added, relies upon the territory scope. The distinctive dynamic hub sending calculations given underneath:

\subsection{VIRTUAL FORCE BASED CALCULATION (VFA)}

It is one of the well-known methodologies for hub sending. In this calculation as critical recognitions the obstructions, sensor hubs and the scope territories are unsurprising to empower appealing or loathsome power inside the hubs can be shaped. In VFA three presumptions are made initial, a single hub inside its correspondence range ought to have the capacity to accomplish relative position of different hubs. Second, just as indicated by the ascertained consequences of the calculation all the left behind hubs will move adequately.

\subsection{VITALITY MINDFUL HUB POSITION IN WSN}

Vitality utilization and abuse of WSN innovation is the key issue for the hub organisation nowadays. Lifetimes of WSN influenced with a few components like MAC outline with vitality productive, topology administration and blunder control systems.

\subsection{NEURAL SYSTEMS}


By copying the association and preparing frameworks of organic nerves. The $\mathrm{NN}$ forms data. It associates an enormous number of neurons: and everyone has a predisposition, and two capacities one is exchange capacity and second is the initiation work. The between neuron association is known as the weight. NN used in a great deal of day by day practices, for example, the back-proliferation neural (BPN) organise generally been used.

\section{NEURAL NETWORK-BASED SCHEMES}

Some of the neural network based schemes that can be used for the wireless sensor networks or other mode of wireless networks can be observed as follows,

Dana conspires: It is a strong preparing confinement approach which is disconnected. The contributions of the system display are comprised of the directions and plausible separations of RSSI of the three grapple hubs, and the yield is arranged. It is a strong, web-based preparing confinement approaches which. HCs between the obscure hubs are the contributions of the system model and all accessible stay hubs, and facilitate the yield. It might yield expansive limitation blunders and neglect to recognize an obscure hub effectively because this plan utilises just the assessed separations of HCs. Some of the other scheme utilized for deployment is given as follows in the following table,

Table 1. Outcome of the Models available

\begin{tabular}{|c|c|c|c|}
\hline S.No. & Year & Technology Used & Outcome \\
\hline 1 & 2013 & $\begin{array}{c}\text { Energy utilization in } \\
\text { communication networks } \\
\text { to be reduced }\end{array}$ & $\begin{array}{c}\text { Network lifetime increased by } \\
\text { reducing the energy utilization }\end{array}$ \\
\hline 2 & 2015 & Node deployment schemes & $\begin{array}{c}\text { Node deployment has done } \\
\text { smoothly, and the performance of } \\
\text { the network analysed with } \\
\text { various performance metrics }\end{array}$ \\
\hline
\end{tabular}

VNBP plot: by arbitrarily creating virtual hubs at sinks to build the quantity of stay hubs VNBP means to show signs of improvement execution when contrasted with past limitation plans. What's more, the virtual hubs delivered with arranges however with no correspondence capacity [6].

\section{CONCLUSIONS}

One of the primary issues in WSNs is Localization. In NLOS which implies the nonviewable pathway which is a sort of situations a flag which is utilised to decide the separation between hubs, given the impediments between the grapple hubs and different hubs, can't go through a straight way. What's more, limitation blunder built on account of the current problems confinement technique based AOA estimation has brilliant execution in restriction in the NLOS conditions. Another neural system based hub restriction plot is utilised to expand the confinement precision and limitation achievement rates. At a reasonable cost, this plan when contrasted with different plans produces higher confinement achievement rates and littler restriction blunders. For improving the great territory scope of the conveyed hubs by abusing hubs motility, A Cluster-Based calculation utilised. An ideal strategy for planning creates by Markov Decision Process show. 


\section{References}

[1] Jaspreet Kaur et.al. "A Review on Deployment schemes in Wireless Sensor Network”, Advances in Computational Sciences and Technology, Vol.10, No.5, pp.1161-1172.

[2] https://www.elprocus.com/introduction-to-wireless-sensor-networks-types-and-applications/ [Last Accessed on 10-05-(2019)]

[3] V. Karthik, "Region-Based Scheduling With Multiple Mobile Robots for Data Collection Strategies in Wireless Sensor Networks", International Journal of Innovative Research in Science, Engineering and Technology, Vol. 2, No.7, July (2013), pp. 3416-3425.

[4] M. Vijayalakshmi, V. Vanitha, "Cluster-Based Adaptive Prediction Scheme for Energy Efficiency In Wireless Sensor Networks", International Conference on Current Trends in Engineering Research, ICCTER-(2014), August, 2014, pp. 10-11.DOI: 10.1049/iet-ifs.2012.0052

[5] Nasser Aghaie, Mohammad Ali Tinati, "Localization of WSN Nodes Based on NLOS Identification Using AOAs Statistical Information”, Iranian Conference on Electrical Engineering (ICEE), (2016), IEEE, pp.496501

[6] Amir EhsaniZonouz, Liudong Xing, Vinod M. Vokkarane, Yan (Lindsay) Sun, "Hybrid wireless sensor networks: reliability, cost and energy-aware approach", IET, The Institution of Engineering and Technology, (2016), pp.1-7. DOI: 10.1049/iet-wss.2014.0131

[7] Renuka .R. Patil, Dr Suresha, "Deployment Algorithms in WSN-A Study”, International Research Journal of Mobile and Wireless Communications, Vol.04, Special Issue, (2013), pp.174-181. DOI: 10.1109/IADCC.2015.7154764

[8] Po-Jen Chuang, Yi-Jun Jiang, "Effective neural network-based node localization scheme for wireless sensor networks", IET Wireless Sensor Syst., Vol. 4, No.2, (2014), pp. 97-103. DOI: 10.1049/iet-wss.2013.0055

[9] Annie UthraRajan, Kasmir Raja S.V, Antony Jeyasekar, Anthony J. Lattanze, "Energy-efficient predictive congestion control for wireless sensor networks”, IET Wireless. Sens. Syst., Vol. 5, No.3, (2015), pp. 115123.DOI: 10.1049/iet-wss.2013.0101

[10] Vijay S. Rao, R. Venkatesha Prasad, Ignas G. M. M. Niemegeers, “Optimal Task Scheduling Policy in Energy Harvesting Wireless Sensor Networks”, IEEE Wireless Communications and Networking Conference (WCNC) 2015, Mar (2015), pp. 1030-1035. DOI: 10.1109/WCNC.2015.7127611

[11] Tiago Semprebomy, Carlos Montez, Gustavo Araujo, Paulo Portugal, "A Sleep-Scheduling Scheme for Enhancing QoS and Network Coverage", in IEEE 802.15.4 WSN", IEEE, (2015), pp.1-4.DOI: 10.1109/WFCS.2015.7160579 
Deployment Schemes in WSNs 